\title{
THE NUMBER OF BLOCKS WITH A GIVEN DEFECT GROUP
}

\author{
LAURENCE BARKER
}

Abstract. Given a $p$-subgroup $P$ of a finite group $G$, we express the number of $p$-blocks of $G$ with defect group $P$ as the $p$-rank of a symmetric integer matrix indexed by the $N(P) / P$-conjugacy classes in $P C(P) / P$. We obtain a combinatorial criterion for $P$ to be a defect group in $G$.

QUESTION A. Given a p-subgroup $P$ of a finite group $G$, how many p-blocks of $G$ have defect group $P$ ? In particular, when is $P$ a defect group in $G$ ?

An answer to this venerable question was given in Robinson [5], reformulated in Broué [2], and generalised and further illuminated in Broué Robinson [3]. The number of $p$-blocks with defect group $P$ is presented, in those three works, as the $p$-rank of a symmetric integer matrix indexed by certain conjugacy classes of $G$.

QUESTION B. Supposing that $G$ is a normal subgroup of a finite group $F$, what is the number $f_{0}(G, F)$ of $F / G$-orbits of defect-zero p-blocks of $G$ whose stabilisers in $F / G$ are $p^{\prime}$-groups?

Question B is more general than Question A because Brauer's extended first main theorem describes a bijective correspondence between the p-blocks of $G$ with defect group $P$, and the $N_{G}(P) / P C_{G}(P)$-orbits of defect-zero $p$ blocks $b$ of $P C_{G}(P) / P$ such that the stabiliser of $b$ in $N_{G}(P) / P C_{G}(P)$ is a $p^{\prime}$-group. In answer to Question $\mathrm{B}$, Theorem 5 below expresses $f_{0}(G, F)$ as the p-rank of a symmetric integer matrix $\Psi(G, F)$ indexed by the $F$-conjugacy classes of $G$. Corollary 6 spells out the new answer thus provided to Question A.

In view of the local reduction indicated above, and also in view of another local reduction conjectured by Alperin [1], the number $f_{0}(G)=f_{0}(G, G)$ of defect-zero $p$-blocks of $G$ is of especial interest. A description of $f_{0}(G)$ as the $p$-rank of a symmetric integer matrix indexed by the conjugacy classes of $G$ has already been given by Robinson [5], but it is to be noted that the matrix $\Psi(G)=\Psi(G, G)$ has the feature of being independent of $p$.

This work is a synthesis of character-theoretic constructions in Strunkov [6] and $G$-algebra-theoretic techniques implicit in Broué [2], Broué-Robinson [3], Robinson [5]. We also make use of a $G$-algebra-theoretic approach to Clifford theory. I am grateful to Robinson for communicating to me an illuminating formulation of material in [6]. 
Let $O$ be a complete local noetherian commutative ring whose residue field $O / J(O)$ has prime characteristic $p$, and whose field of fractions $k$ has characteristic zero. We shall assume that $\kappa$ splits for all the given groups under consideration. When speaking of an integer, we shall always be refering to a rational integer, and we shall identify the integers with the elements of the minimal unital subring of $O$. Also, we shall identify the $p$-blocks of $G$ with the block idempotents of $O G$. Let $\operatorname{Irr}(\kappa G)$ denote the set of irreducible $\kappa G$-characters, and $\operatorname{Irr}_{0}(\kappa G)$ the subset consisting of those $\chi \in \operatorname{Irr}(\kappa G)$ such that $|G| / \chi(1)$ is coprime to $p$.

The conjugation action of $F$ on its normal subgroup $G$ induces algebra automorphisms of $\kappa G$ and $O G$, and induces permutations of $\operatorname{Irr}(\kappa G)$. Recall that, for $H \leqslant K \leqslant F$, the image $(O G)_{H}^{K}$ of the relative trace map $\operatorname{Tr}_{H}^{K}:(O G)^{H} \rightarrow$ $(O G)^{K}$ is an ideal of the $K$-fixed subalgebra $(O G)^{K}$ of $O G$. For each irreducible $\kappa G$-character $\chi$, we write $N_{F}(\chi)$ for the stabiliser of $\chi$ in $F$, and write $b_{\chi}^{F}$ for the primitive idempotent of the commutative algebra $(O G)^{F}$ such that $\chi\left(b_{\chi}^{F}\right)=\chi(1)$. Note that $b_{\chi}^{F}$ is the sum of the $F$-conjugates of the $p$-block $b_{\chi}^{G}$ of $G$ containing $\chi$. We say that a primitive idempotent $b$ of $(O G)^{F}$ is projective provided $b \in(O G)_{1}^{F}$ (when $G=F$, these idempotents are precisely the defectzero $p$-blocks of $G$ ).

Proposition 1. There is a bijective correspondence between the projective primitive idempotents $b$ of $(O G)^{F}$, and the F-orbits of irreducible $\kappa G$-characters $\chi$ such that $\left|N_{F}(\chi)\right| / \chi(1)$ is coprime to $p$, whereby $b$ corresponds to the F-orbit of $\chi$ provided $b=b_{\chi}^{F}$.

Proof. Let $\chi$ be an irreducible $\kappa G$-character, and put $N=N_{F}(\chi)$. It is well-known that $b_{\chi}^{G i}$ is a defect-zero $p$-block of $G$ if, and only if, $|G| / \chi(1)$ is coprime to $p$. When these equivalent conditions hold, the $F$-conjugates of $\chi$ are precisely the irreducible $\kappa G$-characters $\chi^{\prime}$ such that $b_{\chi}^{F}=b_{\chi^{\prime}}^{F}$. So it suffices to prove that $b_{\chi}^{F}$ is projective if, and only if, $|N| / \chi(1)$ is coprime to $p$.

Suppose that $|N| / \chi(1)$ is coprime to $p$. Then $b_{\chi}^{G}$ is a defect-zero block of $O G$, that is, $b_{\chi}^{G}=\operatorname{Tr}_{1}^{G}(\eta)$ for some $\eta \in O G$. Also, $|G: N|$ is coprime to $p$, and $N$ is the stabiliser of $b_{x}^{F}$ in $F$, hence the primitive idempotent

$$
b_{x}^{F}=\operatorname{Tr}_{N}^{F}\left(b_{\chi}^{G}\right)=\operatorname{Tr}_{1}^{F}(\eta /|G: N|)
$$

is projective. Conversely, suppose that $b_{x}^{F}$ is projective. By Mackey decomposition,

$$
(O G)_{\mathrm{i}}^{F} \subseteq(O G)_{1}^{N} \subseteq(O G)_{1}^{G} .
$$

So the idempotent $b_{\chi}^{G}=b_{\chi}^{F} b_{\chi}^{G}$ belongs to the ideal $(O G)_{1}^{N}$ of $(O G)^{N}$, and in particular, $b_{\chi}^{G}$ is a defect-zero block of $O G$. Therefore, $|G| / \chi(1)$ is coprime to $p$, and writing $b_{\chi}^{G}=\operatorname{Tr}_{1}^{N}(\mu)$ for some $\mu \in O G$, we have

$$
b_{\chi}^{G}=\operatorname{Tr}_{1}^{N}\left(\mu b_{\chi}^{G}\right)=|N: G| \operatorname{Tr}_{1}^{G}\left(\mu b_{\chi}^{G}\right)
$$

whereupon $|N: G|$ must be coprime to $p$.

In particular, $f_{0}(G, F)$ is the number of projective primitive idempotents of $(O G)^{F}$. Let $b_{G}^{F}$ be the sum of the projective primitive idempotents of $(O G)^{F}$. 
Thus $b_{G}^{G}$ is the sum of the defect-zero $p$-blocks of $G$. We have a direct sum of free cyclic $O$-modules

$$
(O G)^{F} b_{G}^{F}=\underset{h}{\oplus}(O G)^{F} b
$$

where $b$ runs over the projective primitive idempotents of $(O G)^{F}$. Iizuka Watanabe [4, Lemma 2] proved the following result in the special case $G=F$.

\section{Lemma 2: $(O G)^{F} b_{G}^{F} \subseteq\left((O G)_{1}^{F}\right)^{2} \subseteq(O G)^{F} b_{G}^{F} \oplus J(O)(O G)^{F}\left(1-b_{G}^{F}\right)$}

Proof: Let $V=(O G)^{F} b_{G}^{F}$ and $U=\left((O G)_{1}^{F}\right)^{2}$ as ideals of $(O G)^{F}$. Clearly, $b_{G}^{F} \in V$, so $V \subseteq U$. Now

$$
U=\bigoplus_{\chi \in \epsilon_{F} \operatorname{Irr}(\kappa G)} U b_{\chi}^{F}
$$

where the notation indicates that $\chi$ runs over the $F$-orbits of $\operatorname{Irr}(\kappa G)$. Let us fix an irreducible $\kappa G$-character $\chi$. If $b_{\chi}^{F}$ is projective, then $b_{\chi}^{F} \in V$, so $U b_{\chi}^{F} \subseteq V$. Assuming now that $b_{\chi}^{F}$ is not projective, we have $b_{G}^{F} b_{\chi}^{F}=0$, and it suffices to show that $U b_{\chi}^{F} \subseteq J(O)(O G)^{F}$.

First suppose that $\chi \in \operatorname{Irr}_{0}(\kappa G)$. Then $b_{\chi}^{F} \notin U$, so $U b_{\chi}^{F}$ is strictly contained in the free cyclic $O$-module $(O G)^{F} b_{\chi}^{F}$. Therefore $U b_{\chi}^{F} \subseteq J(O)(O G)^{F}$ in this case. Now suppose that $\chi \notin \operatorname{Irr}_{0}(\kappa G)$. We observed above that $(O G)_{1}^{F} \subseteq(O G)_{1}^{G}$, and that the assertion holds when $G=F$. So

$$
U b_{\chi}^{F} \subseteq U \subseteq\left((O G)_{1}^{G}\right)^{2} \subseteq Z O G \cdot b_{G}^{G} \oplus J(O) \cdot Z O G\left(1-b_{G}^{G}\right) .
$$

But $b_{x}^{G} b_{\chi}^{F}=0$, so $U b_{x}^{F} \subseteq J(O) \cdot Z O G \cap(O G)^{F}=J(O)(O G)^{F}$.

For each irreducible $\kappa G$-character $\chi$, let $e_{\chi}^{F}$ be the primitive idempotent of $(\kappa G)^{F}$ such that $\chi\left(e_{\chi}^{F}\right)=\chi(1)$, and let $\omega_{\chi}^{F}$ be the algebra map $(\kappa G)^{F} \rightarrow \kappa$ such that $\omega\left(e_{\chi}^{F}\right)=1$. Then $\omega_{\chi}^{F}$ is a restriction of the central character $\omega_{\chi}^{G}$ associated with $\chi$. That is to say, $\omega_{\chi}^{F}=\chi / \chi(1)$ on $(\kappa G)^{F}$. Let $\omega_{G}^{F}:(\kappa G)^{F} \rightarrow \kappa$, and $v_{G}^{F}:(\kappa G)^{F} \otimes_{\kappa}(\kappa G)^{F} \rightarrow \kappa$ be the characters afforded by the transiation actions of $(\kappa G)^{F}$ and $(\kappa G)^{F} \otimes_{\kappa}(\kappa G)^{F}$ on $(\kappa G)^{F}$. Thus, given $\zeta, \zeta^{\prime} \in(\kappa G)^{F}$, the trace of the action of $\zeta$ on $(\kappa G)^{F}$ is

$$
\omega_{G}^{F}(\zeta)=\sum_{\chi \in F \mathrm{Irr}(\kappa G)} \omega_{\chi}^{F}(\zeta)
$$

while the trace of the action of $\zeta \otimes \zeta^{\prime}$ on $(\kappa G)^{F}$ is

$$
v_{G}^{F}\left(\zeta \otimes \zeta^{\prime}\right)=\omega_{G}^{F}\left(\zeta \zeta^{\prime}\right)=\sum_{\chi \in \operatorname{Irr}(\kappa G)} \omega_{\chi}^{F}(\zeta) \omega_{\chi}^{F}\left(\zeta^{\prime}\right)
$$

We define linear maps $\varphi_{G}^{F}: \kappa G \rightarrow \kappa$ and $\psi_{G}^{F}: \kappa(G \times G) \rightarrow \kappa$ given by

$$
\begin{gathered}
\varphi_{G}^{F}(g)=|\{(x, w) \in G \times F: g=[x, w]\}|, \\
\psi_{G}^{F}(g, h)=\left|\left\{(x, w, v) \in G \times F \times F: g v h^{-1} v^{-1}=[x, w]\right\}\right|
\end{gathered}
$$

for $g, h \in G$. Here, $[x, w]=x w x^{-1} w^{-1}$. The next result shows that $\varphi_{G}^{F}$ is a $\kappa G$ character, and that $\psi_{G}^{F}$ is a $\kappa(G \times G)$-character. Recall that the irreducible $\kappa(G \times G)$-characters are the $\kappa(G \times G)$-characters of the form $\chi * \chi^{\prime}$ with 
$\chi, \chi^{\prime} \in \operatorname{Irr}(\kappa G)$, where

$$
\left(\chi * \chi^{\prime}\right)(g, h)=\chi(g) \chi^{\prime}\left(h^{-1}\right)
$$

For any element $x \in G$, we write $[x]_{F}$ for the set of $F$-conjugates of $x$. For any subset $A \subseteq G$, we write $A^{+}$for the sum in $\kappa G$ of the elements of $[x]_{F}$. Thus $\left\{[x]_{F}^{\vdash}: x \in G\right\}$ is a $\kappa$-basis for $(\kappa G)^{F}$.

LFMMA 3.

(a) $\phi_{G}^{F}=\sum_{\chi \in \operatorname{lrr}(\kappa G)}\left|N_{r}(\chi)\right| \chi / \chi(1)$.

(b) $\psi_{G}^{F}=\sum_{\chi \in \operatorname{Irr}(\kappa G), v N_{F}(\chi) \subseteq F}\left(\left|N_{F}(\chi)\right| / \chi(1)\right)^{2} \chi *{ }^{v} \chi$.

Proof. Given an element $\zeta \in(\kappa G)^{F}$, and writing

$$
\zeta[x]_{F}^{+}=\sum_{y \in r, G} \zeta_{x, y}[y]_{F}^{+}
$$

then $\omega_{G}^{F}(\zeta)=\sum_{y \in R} \zeta_{1 . .}$, where the notation indicates that the two sums are indexed by representatives $y$ of the $F$-conjugacy classes of $G$. So for $g, h \in G$, we have

$$
\begin{aligned}
& \left.\psi_{G}^{F}(g, h)=\mid\{x, w, v, u) \in G \times F \times F \times F: u g u^{-1} v h^{-1} v^{-1} w x w^{-1}=x\right\}|/| F \mid \\
& =\sum_{x \in F G}\left|\left\{(w, v, u) \in F \times F \times F: u g u^{-1} v h^{-1} v^{-1} w x w^{-1}=x\right\}\right| /\left|C_{F}(x)\right| \\
& \left.=\sum_{x \in F G} \mid\{y, v, u) \in[x]_{F} \times F \times F: u g u^{-1} v h^{-1} v^{-1} y=x\right\} \mid \\
& =v_{G}^{F}\left(\operatorname{Tr}_{1}^{F}(g) \otimes \operatorname{Tr}_{1}^{F}\left(h^{-1}\right)\right) \\
& =\sum_{\chi \in F, \operatorname{lir}(\kappa(G)} \chi\left(\operatorname{Tr}_{1}^{F}(g)\right) \chi\left(\operatorname{Tr}_{1}^{F}\left(h^{-1}\right)\right) / \chi(1)^{2} \\
& =|G|^{2} \sum_{\chi \in F \operatorname{srr}\left(n^{-} G\right), u G \subseteq F, v G \subseteq F} \chi\left(u g u^{-1}\right) \chi\left(v h^{-1} v^{-1}\right) / \chi(1)^{2} \\
& =\sum_{\chi \in F \operatorname{Irr}(\kappa G), v N_{F}(\chi) \subseteq F}\left(\left|N_{F}(\chi)\right| / \chi(1)\right)^{2} \chi\left(u g u^{-1}\right) \chi\left(\mathrm{vh}^{-1} \mathrm{v}^{-1}\right) .
\end{aligned}
$$

Part (b) is thus estabiished. Part (a) may be proved either by showing, similarly, that

$$
\varphi_{G}^{F}(g)=\omega_{G}^{F}\left(\operatorname{Tr}_{1}^{F}(g)\right)=\sum_{\chi \in \operatorname{Irr}(\kappa G)}\left|N_{F}(\chi)\right| \chi(g) / \chi(1)
$$

or else by observing that $\varphi_{G}^{F}(g)=\psi_{G}^{F}(g, 1) /|F|$.

Lemma 4. Given $g, h \in G$, then:

(a) $\left|C_{F}(g)\right|$ divides $\varphi_{G}^{F}(g)$;

(b) $\left.\mid C_{F}\right)(g)|| C_{F}(h) \mid$ divides $\psi_{G}^{F}(g, h)$.

Proof. The proof of Lemma 3 shows that $\varphi_{G}^{F}(g) /\left|C_{F}(g)\right|=\omega_{G}^{F}\left([g]_{F}^{+}\right)$, and $\psi_{G}^{F}(g, h) /\left|C_{F}(g)\right|\left|C_{F}(h)\right|=v_{G}^{F}\left([g]_{F}^{+} \otimes\left[h^{-1}\right]_{F}^{+}\right)$. Any rational number belonging to $O$ must be an integer. 
We define a symmetric integer matrix $\Psi(G, F)=\left(\psi_{G}^{F}(g, h)\right)_{g, h \in, G}$ (indexed by representatives of the $F$-conjugacy classes in $G)$. For any integer matrix $\Psi$, let $\operatorname{rk}_{p} \Psi$ denote the $p$-rank of $\Psi$ (the rank of the reduction of $\Psi$ modulo $p$ ).

TheOREM 5. (a) $f_{0}(G, F)=\mathrm{rk}_{p} \Psi(G, F)$.

(b) $(O G)^{F}$ has a projective primitive idempotent if, and only if, $\varphi_{G}^{F}(x)$ is coprime to $p$ for some $x \in G$.

Proof. Any projective primitive idempotent $b$ of $(O G)^{F}$ may be written in the form $b=\operatorname{Tr}_{1}^{F}\left(\sum_{\mathrm{g} \in G} b_{g} g\right)$ with each $b_{g} \in O$. Given another projective primitive idempotent $h^{\prime}$ of $(O G)^{F}$, the proof of Lemma 3 gives

$$
\psi_{G}^{F}\left(\sum_{g \in G} b_{g} g \otimes \sum_{h \in G} b_{h}^{\prime} h^{-1}\right)=v_{G}^{F}\left(b \otimes b^{\prime}\right)=\omega_{G ;}^{F}\left(b h^{\prime}\right)
$$

which is zero when $b \neq b^{\prime}$. But $\omega_{G}^{F}(b)=1$ because $b=e_{\chi}^{F}$ for some $\chi \in \operatorname{Ir}_{f_{1}}(\kappa G)$. So $f_{0}(G, F) \leqslant \mathrm{rk}_{p} \Psi_{G}^{F}$. The proof of Lemma 3 also shows that

$$
\psi_{G}^{F}(g, h)=\omega_{G}^{F}\left(\operatorname{Tr}_{1}^{F}(g) \operatorname{Tr}_{1}^{F}\left(h^{-1}\right)\right)
$$

for $g, h \in G$. But $f_{0}(G, F)$ is the $O$-rank of $(O G)^{F} b_{G}^{F}$, so Lemma 2 forces $f_{0}(G, F) \geqslant \operatorname{rk}_{p} \Psi_{G}^{F}$, establishing part (a).

Part (b) is the assertion that $\psi_{G}^{F}(g, h)$ is coprime to $p$ for some $g, h \in G$ if and only if $\varphi_{G}^{F}(x)$ is coprime to $p$ for some $x \in G$. The forwards implication holds because $\psi_{G}^{F}(g, h)=\varphi_{G}^{F}\left(g \operatorname{Tr}_{1}^{F}\left(h^{-1}\right)\right)$ for all $g, h \in G$. Proposition 1 and Lemma 3(a) give the reverse implication.

Thanks to Lemma 4(b), $f_{0}(G, F)$ is, in fact, the $p$-rank of the submatrix of $\Psi(G, F)$ indexed by those representatives $g$ such that $C_{F}(g)$ is a $p^{\prime}$-group.

Corollary 6. Let $P$ be a p-subgroup of $G$. Define $\bar{C}=P C_{G}(P) / P$ and $\bar{N}=N_{G}(P) / P$. Then:

(a) the number of p-blocks of $G$ with defect group $P$ is $\mathrm{rk}_{p} \Psi(\bar{C}, \bar{N})$;

(b) $P$ is a defect group of a $p$-block of $G$ if, and only if, there exists some $g \in \bar{C}$ such that $p$ does not divide the number of solutions in $x \in \bar{C}$ and $w \in \bar{N}$ to the equation $g=[x, w]$.

Proof. This is immediate from Proposition 1, Theorem 5, and Brauer's extended first main theorem.

Putting $G=F$ in Theorem 5, or $P=1$ in Corollary 6 , we deduce that $f_{0}(G)$ is the $p$-rank of the symmetric integer matrix $\Psi(G):=\Psi(G, G)$ indexed by the representatives of the conjugacy classes of $G$. We note, as above, that to calculate $f_{0}(G)$, we need only consider the submatrix indexed by the representatives of the defect-zero conjugacy classes. Furthermore, we recover the special case of Strunkov [6, Theorem 1] asserting that $G$ has a defect-zero $p$-block if, and only if, there exists an element $g \in G$ such that $p$ does not divide the number of ways of expressing $g$ as a commutator $[x, w]$ with $x, w \in G$. 
We end by observing another relationship between the character $\varphi_{G}=\varphi_{G}^{G}$ and the number of defect-zero $p$-blocks of $G$. Let us fix a Sylow $p$-subgroup $S$ of $G$. We write $\left(\operatorname{Res}_{S}^{G}\left(\varphi_{G}\right), 1_{S}\right)$ to denote the multiplicity of the trivial $\kappa S$ module in the restriction of $\varphi_{G}$ to $S$. Note that

$$
\left(\operatorname{Res}_{S}^{G}\left(\varphi_{G}\right), \mathbf{1}_{S}\right)=|\{(x, w) \in G \times G:[x, w] \in S\}| /|S| .
$$

Proposition 7. Modulo $p$, we have a congruence

$$
|G: S| f_{0}(G) \equiv_{p}\left(\operatorname{Res}_{S}^{G}\left(\varphi_{G}\right), 1_{s}\right) .
$$

Proof. For any $\zeta \in Z O G$, Lemma 3(a) gives

$$
\varphi_{G}(\zeta)=|G| \sum_{\chi \in \operatorname{Irr}(\kappa G)} \omega_{\chi}(\zeta)
$$

In particular, $|G|$ divides $\psi_{G}(\zeta)$. Let $G_{p}$ denote the set $p$-elements of $G$. By Iizuka Watanabe [4, Lemmas 3 and 4], if $\chi \in \operatorname{Irr}_{0}(\kappa G)$ then $\omega_{\chi}\left(G_{p}^{+}\right)=1$, otherwise $p$ divides $\omega_{\chi}\left(G_{n}^{+}\right)$. So

$$
f_{0}(G) \equiv{ }_{p} \varphi_{G}\left(G_{p}^{+}\right) /|G| .
$$

Let $N=N_{G}(S)$. A well-known variant of Sylow's theorem asserts that the number of Sylow $p$-subgroups of $G$ containing any given $p$-subgroup is congruent to unity modulo $p$. Applying this to the cyclic $p$-subgroups, we deduce that $|G: N|$ is congruent to unity modulo $p$, and so too is the coefficient in $\operatorname{Tr}_{N}^{G}\left(\mathrm{~S}^{+}\right)$of each $p$-element. Hence $\left(\operatorname{Tr}_{N}^{G}\left(S^{+}\right)-G_{p}^{\mp p}\right) / p \in Z O G$, and $p|G|$ divides $\varphi_{G}\left(\operatorname{Tr}_{N}^{G}\left(S^{+}\right)-G_{r}^{+}\right)$. Therefore

$$
\varphi_{G}\left(G_{p}^{+}\right) /|S| \equiv_{p} \varphi_{G}\left(\operatorname{Tr}_{N}^{G}\left(S^{+}\right)\right) /|S| \equiv_{n} \varphi_{G}\left(S^{+}\right) /|S|=\left(\operatorname{Res}_{S}^{G}\left(\varphi_{G}\right), 1_{S}\right) .
$$

\section{References}

1. J. L. Alperin. Weights for finite groups. Proc. Symp. Pure Math., 47 (1987), 369379.

2. M. Broué. On a theorem of G. Robinson. J. London Math. Soc., 29 (1984), 425-434.

3. M. Brous and G. R. Robinson. Bilinear forms on $G$-algebras. J. Algebra, 104 (1986), 377-396.

4. K. lizuka and A. Watanabe. On the number of blocks of irreducible characters of a finite group with a given defect yroup. Kumamoto J. Sci. (Math.), 9 (1973), 5561.

5. G. R. Robinson. The number of blocks with a given defect group. J. Algebra, 84 (1983), 493 502.

6. S. P. Strunkov. Existence and the number of $p$-blocks of defect 0 in finite groups. Algebra and Logic. 30 (1992), 231241.

Dr. L. J. Barker.

Department of Mathematics,

Bilkent University,

06533 Bilkent, Ankara,

Turkey.
20C20: GROUP THEORY AND GENERALIZATIONS: Represen- tation theory of groups: Modular rep- resentations and characters.
Received on the 6th of September, 1996.

